\title{
Finite Groups Having the Same Prime Graph As the Group $\operatorname{Aut}\left(J_{2}\right)$
}

\author{
A. S. Kondrat'ev ${ }^{1}$
}

Received February 12, 2012

\begin{abstract}
Finite groups having the same prime graph as the group $\operatorname{Aut}\left(J_{2}\right)$ are described. This solves the problem posed by B. Khosravi.

Keywords: finite group, group Aut $\left(J_{2}\right)$, prime graph, recognition by prime graph.
\end{abstract}

DOI: $10.1134 / \mathrm{S} 0081543813090071$

\section{INTRODUCTION}

Let $G$ be a finite group. Denote by $\pi(G)$ the set of prime divisors of the order of $G$, and denote by $\omega(G)$ the spectrum of $G$, i.e., the set of all orders of its elements. The set $\omega(G)$ defines the prime graph (the Gruenberg-Kegel graph) $\Gamma(G)$ of the group $G$, where the vertex set is $\pi(G)$ and two different vertices $p$ and $q$ are adjacent if and only if $p q \in \omega(G)$. The number of connected components of the graph $\Gamma(G)$ is denoted by $s(G)$, and the set of its connected components is denoted by $\left\{\pi_{i}(G) \mid 1 \leq i \leq s(G)\right\}$. For a group $G$ of even order, we assume that $2 \in \pi_{1}(G)$.

A group $G$ is called recognizable (by spectrum) if any finite group $H$ satisfying the condition $\omega(H)=\omega(G)$ is isomorphic to $G$. Recognition of finite groups by spectrum is a well-developed direction of research (see Mazurov's survey [6]), and a closely connected research area is recognition of finite groups by their prime graph. A group $G$ is called recognizable by prime graph if, for any finite group $H$, the equality $\Gamma(H)=\Gamma(G)$ of graphs implies the isomorphism $H \cong G$ of these groups. Here, the equality of the graphs $\Gamma(H)$ and $\Gamma(G)$ means the coincidence of their vertex sets and edge sets, respectively. It is clear that the recognizability of a finite group by prime graph implies its recognizability by spectrum.

The proof of the recognizability of any finite simple group by its order and spectrum has been completed recently (see [1]). The conjecture that finite simple groups are, as a rule, recognizable by their order and prime graph seems to be quite plausible. For many finite simple groups, this conjecture has been verified. For sporadic simple groups, this was done in 1996 by Chen [11].

In 2003, Hagie [13] gave the first examples of finite groups recognizable by prime graph, namely, the sporadic simple groups $J_{1}, M_{22}, M_{23}, M_{24}$, and $C_{2}$. In the same paper, a description (but not a complete classification) of finite groups $G$ such that $\Gamma(G)=\Gamma(S)$, where $S$ is a sporadic simple group, was obtained. The results of [3, Theorems 5, 6] on the sporadic groups $M_{11}, M_{12}$, and $J_{2}$, essentially refine Hagie's corresponding results.

\footnotetext{
${ }^{1}$ Institute of Mathematics and Mechanics, Ural Branch of the Russian Academy of Sciences, ul. S. Kovalevskoi 16, Yekaterinburg, 620990 Russia; Institute of Mathematics and Computer Science, Ural Federal University, pr. Lenina 51, Yekaterinburg, 620000 Russia email: A.S.Kondratiev@imm.uran.ru
} 
Khosravi [16-18] obtained similar results for automorphism groups of all finite sporadic simple groups except for $\operatorname{Aut}\left(J_{2}\right)$ and posed the problem of describing the structure of finite groups $G$ such that $\Gamma(G)=\Gamma\left(\operatorname{Aut}\left(J_{2}\right)\right)$. If $S$ is a sporadic simple group, then $|\operatorname{Aut}(S): \operatorname{Inn}(S)| \leq 2$ and the graphs $\Gamma(S)$ and $\Gamma(\operatorname{Aut}(S))$ are disconnected except for the graphs $\Gamma\left(\operatorname{Aut}\left(J_{2}\right)\right)$ and $\Gamma(\operatorname{Aut}(M c L))$.

In the present paper, Khosravi's problem is solved. The following theorem is proved.

Theorem. Suppose that $G$ is a finite group and $\Gamma(G)=\Gamma\left(\operatorname{Aut}\left(J_{2}\right)\right)$. Then, one of the following statements holds:

(1) $G$ is solvable, a 2-complement in $G$ is a Frobenius group whose kernel is a 7-group and a complement is a cyclic $\{3,5\}$-group $B$ of order divisible by 15 , and the quotient group $G / O_{\{2,7\}}(G)$ is isomorphic to a subgroup from $\operatorname{Hol}(B)$ of order dividing $8|B|$;

(2) $G$ is solvable; a 2-complement $R$ in $G$ is a Frobenius group of the form $A \lambda B$, where $A=F(R)$ is a $\{3,5\}$-group of order divisible by 15 and $B$ is a cyclic 7 -group; the quotient group $O_{7^{\prime}}(G) / O_{2}(G)$ has normal 2-complement $F(R) O_{2}(G) / O_{2}(G)$; and the quotient group $G / O_{7^{\prime}}(G)$ is isomorphic to $B$ or to the dihedral group of order $2|B|$;

(3) $G$ is solvable; a 2-complement $R$ in $G$ is a 2-Frobenius group of the form $A \lambda B \lambda C$, where $A=F(R)$ is a $\{3,5\}$-group of order divisible by $5, B$ is a cyclic 7-group, and $|C|=3$; the quotient group $\mathrm{O}_{7^{\prime}}(G) / \mathrm{O}_{2}(G)$ has normal 2-complement $F(R) \mathrm{O}_{2}(G) / \mathrm{O}_{2}(G)$; and the quotient group $G / O_{7^{\prime}}(G)$ is isomorphic to a Frobenius group of order $3|B|$ or $6|B|$;

(4) $G / O_{2}(G)$ is isomorphic to one of the groups $A_{8}, S_{8}, A_{9}, S_{9}, S_{6}(2), O_{8}^{+}(2), O_{8}^{+}(2): 2, J_{2}$, or $\operatorname{Aut}\left(J_{2}\right)$;

(5) $G / O_{2}(G)$ is isomorphic to an extension of a nontrivial nilpotent $\{3,5\}$-group $A$ by a group $B$ such that $F^{*}(B)=O_{2}(B) \times L$, where the group $L$ is isomorphic to $A_{7}$, the group $B / O_{2}(B)$ is isomorphic to $A_{7}$ or $S_{7}$, and the group $L$ induces (by conjugation) the irreducible 6-dimensional $G F(p) A_{7}$-module on any $p$-chief factor of the group $\left(G / O_{2}(G)\right)^{\infty}$ for $p \in\{3,5\}$;

(6) $G / O_{2}(G)$ is isomorphic to an extension of a nilpotent $\{3,5\}$-group $A$ of order divisible by 5 by a group $B$ such that $F^{*}(B)=O_{2}(B) \times L$, where the group $L$ is isomorphic to $U_{3}(3)$, the group $B / O_{2}(B)$ is isomorphic to $U_{3}(3)$ or $G_{2}(2)$, $L$ induces on any 3-chief factor of the group $\left(G / O_{2}(G)\right)^{\infty}$ the natural unitary 3-dimensional $G F(9) U_{3}(3)$-module or 6-dimensional irreducible $G F(9) U_{3}(3)$-module, and $L$ induces the 6-dimensional absolutely irreducible $\left.G F(5)\right) U_{3}(3)$-module on any 5 -chief factor of the group $\left(G / O_{2}(G)\right)^{\infty}$;

(7) $G / O_{2}(G)$ is isomorphic to an extension of a nilpotent $\{3,5\}$-group $A$ of order divisible by 5 by a group $B$ such that $F^{*}(B)=O_{2}(B) \times L$, where the group $L$ is isomorphic to $L_{2}(7)$, the group $B / O_{2}(B)$ is isomorphic to $L_{2}(7)$ or $P G L_{2}(7)$, and the group $L$ induces on any $p$-chief factor of the group $\left(G / O_{2}(G)\right)^{\infty}$ the 3-dimensional irreducible $G F\left(p^{2}\right) L_{2}(7)$-module or 6 -dimensional absolutely irreducible $G F(p) L_{2}(7)$-module for $p \in\{3,5\}$;

(8) $G / O_{2}(G)$ is isomorphic to a semidirect product of a nontrivial abelian 3-group $A$ and a group $B$ such that $F^{*}(B)=O_{2}(B) \circ L$, where the group $L$ is isomorphic to $2 \cdot L_{3}(4)$ or $2 \cdot U_{4}(3)$, the group $B / F^{*}(B)$ is isomorphic to a subgroup from $D_{8}$, the involution from $Z(L)$ inverts $A$, and the group $L$ induces the faithful irreducible 6-dimensional $G F(3) L$-module on any 3-chief factor of $A L$;

(9) $G / O_{2}(G)$ is isomorphic to a semidirect product of a nontrivial abelian 3-group $A$ and a group $B$ such that $F^{*}(B)=O_{2}(B) \circ L$, where the group $L$ is isomorphic to $2^{2 \cdot} L_{3}(4)$, the group $B / F^{*}(B)$ is isomorphic to a subgroup from $2^{2}, Z(L)$ is generated by involutions $z_{1}$ and $z_{2}$ such that $A=C_{A}\left(z_{1}\right) \times C_{A}\left(z_{2}\right)$, and $L$ induces the faithful irreducible 6 -dimensional $G F(3) 2 L_{3}(4)$-module on any 3 -chief factor of the group $A L$;

(10) $G / O_{2}(G)$ is isomorphic to a semidirect product of an abelian $\{3,5\}$-group $A$ and a group $B$ 
such that $F^{*}(B)=O_{2}(B) \circ L$, where the group $L$ is isomorphic to $2 J_{2}$, the group $B / O_{2}(B)$ is isomorphic to $J_{2}$ or $\operatorname{Aut}\left(J_{2}\right)$, the involution from $Z(L)$ inverts $A$, the group $L$ induces the faithful irreducible 6-dimensional GF(9)L-module on any 3-chief factor of the group $A L$, and $L$ induces the faithful irreducible 6-dimensional GF(5)L-module on any 5-chief factor of $A L$;

(11) $G / O_{2}(G)$ is isomorphic to an extension of a nilpotent $\{3,5\}$-group $A$ of order divisible by 5 by a group $B$ such that $F^{*}(B)=O_{2}(B) \circ L$, where the group $L$ is isomorphic to $S L_{2}(7)$, the group $B / O_{2}(B)$ is isomorphic to $L_{2}(7)$ or $P G L_{2}(7)$, and $L$ induces on any p-chief factor of the group $\left(G / \mathrm{O}_{2}(G)\right)^{\infty}$ for $p \in\{3,5\}$ either an unfaithful irreducible L-module with kernel of order 2 (see statement $(7)$ ) or the faithful 6-dimensional irreducible $G F\left(p^{2}\right) L$-module.

Each of statements (1)-(11) of the theorem is realized.

Remark. Groups of smallest orders from statements (1)-(3) of the theorem have the form $\left.2 \times\left(7^{4}: 15\right), 2 \times\left(\left(3^{6} \times 5^{6}\right): 7\right)\right)$, and $2 \times\left(\left(5^{6}: 7\right): 3\right)$, respectively.

Corollary. A finite group with the same order and prime graph as in the group $\operatorname{Aut}\left(J_{2}\right)$ is isomorphic to $\operatorname{Aut}\left(J_{2}\right), 2 \times J_{2}$, or $\widehat{J}_{2}$.

Our notation and terminology are mostly standard and can be found in $[4,7-9,15]$. Denote by $G^{\infty}$ the last term in the series of commutator subgroups of a finite group $G$. A splittable extension of a group $A$ by a group $B$ is denoted by $A \lambda B$ or $A: B$. A nonsplittable extension of $A$ by $B$ is denoted by $A \cdot B$. If $n$ is a positive integer and $p$ is a prime, then, as in [9], we denote by $n$ the cyclic group of order $n$ and by $p^{n}$ the elementary abelian group of order $p^{n}$.

If a group $G$ acts on a group or a module $H$, then a nontrivial element $g \in G$ is said to act on $H$ freely (or without fixed points) if $C_{H}(g)=1$.

\section{AUXILIARY RESULTS}

Here, we present some results used in the proof of the theorem.

Proposition 1.1 (Gruenberg-Kegel theorem [20, Theorem A]). If $G$ is a finite group with disconnected prime graph, then one of the following statements holds:

(1) $G$ is a Frobenius group;

(2) $G$ is a 2-Frobenius group;

(3) $G$ is an extension of a nilpotent $\pi_{1}(G)$-group by a group $A$, where $\operatorname{Inn}(P) \leq A \leq \operatorname{Aut}(P)$, $P$ is a simple nonabelian group with $s(G) \leq s(P)$, and $A / \operatorname{Inn}(P)$ is a $\pi_{1}(G)$-group.

The following useful result is well known (see, for example, [2, Lemma 4]).

Proposition 1.2. Suppose that $G$ is a finite quasi-simple group, $F$ is a field of characteristic $p>0, V$ is a faithful absolutely irreducible FG-module, and $\beta$ is a Brauer character of the module $V$. If $g$ is an element from $G$ of prime order coprime with $p|Z(G)|$, then

$$
\operatorname{dim} C_{V}(g)=\left(\left.\beta\right|_{\langle g\rangle},\left.1\right|_{\langle g\rangle}\right)=\frac{1}{|g|} \sum_{x \in\langle g\rangle} \beta(x) .
$$

The following result, which belongs to Mazurov [5, Lemma 1], is often used for solving the problem of recognition of finite groups by spectrum or prime graph.

Proposition 1.3. Suppose that $G$ is a finite group, $N$ is a normal subgroup in $G$, and $G / N$ is a Frobenius group with kernel $F$ and cyclic complement $C$. If $(|F|,|N|)=1$ and $F$ is not contained in $N C_{G}(N) / N$, then $s|C| \in \omega(G)$ for some $s \in \pi(N)$.

We obtain the following analog of Proposition 1.3, which is of independent interest.

$$
\text { PROCEEDings OF The STEKLOV InSTITUTE OF MAThematics } \quad \text { Vol. } 283 \quad \text { Suppl. } 1 \quad 2013
$$


Proposition 1.4. Suppose that $q$ is an odd prime, $q-1$ is not a power of 2 , and $G$ is a finite group of the form $G=P \lambda(T \lambda\langle x\rangle)$, where $P$ is a nontrivial $\{2, q\}^{\prime}$-group, $T$ is a 2-group, $|x|=q$, and $C_{G}(P)=Z(P)$. If $[T, x] \neq 1$, then $C_{P}(x) \neq 1$.

Proof. Let $G$ be a counterexample of minimal order to the proposition. Then, $P$ is a $p$ group for some prime $p$. In view of $[12$, Theorems $5.3 .5,5.3 .6]$, we have $T=C_{T}(x)[T, x]$ and $[T, x]=[T, x, x]$; hence, $T=[T, x] \neq 1$. Since $T\langle x\rangle$ acts faithfully on $P / \Phi(P), P$ is an elementary abelian $p$-group. By [12, Theorem 5.3.8], the group $T$ has an $\langle x\rangle$-invariant special subgroup $E$ such that $\langle x\rangle$ acts nontrivially and irreducibly on $E / \Phi(E)$ and trivially on $\Phi(E)$. Therefore, $T=E$.

Assume that $\Phi(T)=1$. Then, $T\langle x\rangle$ is a Frobenius group. By Proposition 1.3, we have $C_{P}(x) \neq 1$, which contradicts the assumption.

Thus, $T^{\prime}=\Phi(T)=Z(T)$ is a nontrivial elementary abelian 2-group. If $|Z(T)|=2$, then $T$ is an extraspecial 2-group; hence, in view of [8, Property 36.1], we have $C_{P}(x) \neq 1$, which contradicts the assumption.

Thus, $\Phi(T)=Z(T\langle x\rangle)$ is a noncyclic elementary abelian 2-group. Since $p$ does not divide $|T\langle x\rangle|$, by Maschke's theorem (see [12, Theorem 3.3.1]), we have $P=P_{1} \times \ldots \times P_{n}$, where any subgroup $P_{i}$ can be considered as an irreducible $G F(p) T\langle x\rangle$-module. Since $C_{T}(P)=1$, we can assume that $C_{\Phi(T)}\left(P_{1}\right)<\Phi(T)$. If $C_{T}\left(P_{1}\right) \not \leq \Phi(T)$, then $C_{T}\left(P_{1}\right)$ is a nontrivial normal subgroup in $T\langle x\rangle$ and, consequently, $T=\Phi(T) C_{T}\left(P_{1}\right)=C_{T}\left(P_{1}\right)$, a contradiction. Therefore, $C_{T}\left(P_{1}\right)<\Phi(T)$. In view of the minimality of $G$, we have $P=P_{1}$ and $C_{T}\left(P_{1}\right)=1$. Since $\Phi(T)$ is a noncyclic elementary abelian 2-group, there exists an involution $t$ in $\Phi(T)$ such that $C_{P}(t) \neq 1$. Then, however, $C_{P}(t)$ is a proper subgroup of $P$ normal in $G$, which contradicts the irreducibility of the $G F(p) T\langle x\rangle$-module $P$. The proposition is proved.

\section{PROOF OF THE THEOREM}

Lemma 2.1. The graph $\Gamma\left(\operatorname{Aut}\left(J_{2}\right)\right)$ has the form

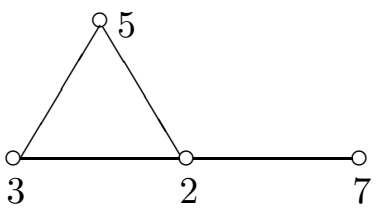

Proof. The statement of the lemma follows from [9].

Lemma 2.2. Let $G$ be a finite simple nonabelian group, and let $\Gamma(G)$ be a subgraph of $\Gamma\left(\operatorname{Aut}\left(J_{2}\right)\right)$. Then, $G$ is isomorphic to one of the groups $A_{5}, L_{2}(7), A_{6}, L_{2}(8), U_{3}(3), U_{4}(2)$, $A_{7}, A_{8}, A_{9}, L_{2}(49), L_{3}(4), U_{3}(5), U_{4}(3), S_{6}(2), O_{8}^{+}(2)$, or $J_{2}$.

Proof. The statement of the lemma follows from Lemma 2.1 and $[9,10,14,19]$. The lemma is proved.

Suppose that $G$ is a finite group and $\Gamma(G)=\Gamma\left(\operatorname{Aut}\left(J_{2}\right)\right)$. In particular, $G$ is a $\{2,3,5,7\}$-group.

Suppose first that $G$ is solvable and $R$ is a 2-complement in $G$. Then, by Lemma 2.1, the graph $\Gamma(R)$ is disconnected; consequently, by Proposition 1.1, the subgroup $R$ is either a Frobenius group or a 2-Frobenius group. Define $\pi=\{3,5\}$. In view of the known properties of finite Frobenius groups, we can assume that $\pi_{1}(R)=\pi$ and $\pi_{2}(R)=\{7\}$.

Let $R$ be a Frobenius group with kernel $A$ and complement $B$.

Assume that $7 \in \pi(A)$. Then, $A$ is a 7 -group and $B$ is a $\pi$-group of order divisible by 15 . It is easy to see that $B$ is a cyclic $\pi$-Hall subgroup in $G$ and $O_{\pi}(G)=1$. In view of [12, Theorem 6.3.2], we have $C_{G}(B) \leq O_{\pi^{\prime}, \pi}(G)$ and $O_{\pi^{\prime}, \pi}(G)=O_{\pi^{\prime}}(G) B$, which verifies statement (1) of the theorem. 
Assume that $7 \in \pi(B)$. Then, $A$ is a nilpotent $\pi$-group of order divisible by 15 and $B$ is a cyclic 7-Hall subgroup in $G$. Set $\tilde{G}=G / O_{2}(G)$ and $K=O_{7^{\prime}}(G)$. Then, $1 \neq O(\tilde{K})=O_{\pi}(\tilde{K})=F(\tilde{K}) \leq \tilde{A}$ and, moreover, $C_{\tilde{K}}(F(\tilde{K}))=Z(F(\tilde{K}))$. Let us show that the group $\tilde{K}$ is $2^{\prime}$-closed. Assume the contrary. In this case, $O(\tilde{K})<O_{2^{\prime}, 2}(\tilde{K})<O_{2^{\prime}, 2,2^{\prime}}(\tilde{K})$. Let $\tilde{T}$ be a (nontrivial) Sylow 2-subgroup in $O_{2^{\prime}, 2}(\tilde{K})$. Then, in view of [12, Theorem 6.3.2], $C_{\tilde{K}}(\tilde{T}) \leq O_{2^{\prime}, 2}(\tilde{K})$. By Frattini's argument, $\tilde{G}=O(\tilde{K}) N_{\tilde{G}}(\tilde{T})$; hence, there exists an element $\tilde{x}$ of order 7 in $N_{\tilde{G}}(\tilde{T})$. Applying Proposition 1.4 to the group $O(\tilde{K}) \lambda(\tilde{T} \lambda\langle x\rangle)$, we find that $\tilde{T}$ centralizes the element $\tilde{x}$. Then, the element $\tilde{x}$ acts identically on the quotient group $\tilde{K} / O_{2^{\prime}, 2}(\tilde{K})$ and, hence, centralizes an element of order 3 or 5 from $\tilde{K}$, which contradicts Lemma 2.1. Thus, the group $\tilde{K}$ is $2^{\prime}$-closed. In view of [12, Theorem 6.3.2], we have $C_{\tilde{G}}(B) \leq K B$, which verifies statement (2) of the theorem.

Let $R$ be a 2-Frobenius group of the form $R=A \lambda(B \lambda C)$. It is clear that $B$ is a cyclic 7-group; consequently, $|C|=3$ and $A$ is a nilpotent $\pi$-group. Arguing as in the preceding paragraph, we verify statement $(3)$ of the theorem.

Suppose that the group $G$ is unsolvable, $S=S(G)$ is its largest solvable normal subgroup, and $\bar{G}=G / S$. It is clear that nonabelian composition factors of $\bar{G}$ are isomorphic to some simple groups from the conclusion of Lemma 2.2.

Lemma 2.3. The order of the group $S$ is not a multiple of 7 .

Proof. Assume that $7 \in \pi(S)$ and $P \in S y l_{7}(S)$. By Frattini's argument, $G=S N_{G}(P)$.

Assume that $N_{G}(P)$ contains an elementary subgroup $V$ of order 9 or 25 . Then, $C_{P}(v) \neq 1$ for some nontrivial element $v \in V$; consequently, $\omega(G)$ contains 21 or 35, which contradicts Lemma 2.1.

Thus, Sylow 3 - and 5-subgroups in the group $\bar{G}$ are cyclic. Then, by Lemma $2.2, \bar{G}$ is almost simple and its socle is isomorphic to one of the groups $A_{5}, L_{2}(7), L_{2}(8)$, or $L_{2}(49)$. The group $\bar{G}$ does not contain an element of order 15 but the group $G$ contains such an element; hence, $\pi(S)$ contains a number $q \in\{3,5\}$. Suppose that $R$ is a $\{q, 7\}$-Hall subgroup in $S$ containing $P$ and $Q \in \operatorname{Syl}_{q}(R)$. Since the group $R$ is solvable and the graph $\Gamma(R)$ is disconnected, we conclude by the Gruenberg-Kegel theorem that either $R$ is a Frobenius group with kernel $P$ and complement $Q$ or $q=3, R$ is a 2-Frobenius group, and $F(R)=O_{3}(R)$. Hence, at least one of the subgroups $P$ and $Q$ is cyclic.

Assume that $P$ is cyclic. Since the group $\operatorname{Aut}(P)$ is abelian in this case, $\overline{C_{G}(P)}$ contains the socle of $\bar{G}$ and, hence, there exists an element of order 3 in $C_{G}(P)$, which is impossible.

Thus, $Q$ is cyclic. Hence, $R$ is a Frobenius group with kernel $P$ and complement $Q$. If 7 divides the order of $\bar{G}$, then, arguing as in the previous paragraph, we conclude that there exists an element of order 7 in $C_{G}(Q)$, which is impossible. Therefore, the group $\bar{G}$ is isomorphic to $A_{5}$ or $S_{5}$.

Let $N=N_{G}(R)$. Since $\{q, 7\}$-Hall subgroups in the solvable group $S$ are conjugate (see [12, Theorem 6.4.1]), we have $G=S N$ and, consequently, $N /(N \cap S) \cong \bar{G}$. As above, we show that $C_{N}(Q)(N \cap S)$ contains the socle of the group $N /(N \cap S)$. Therefore, the group $C_{N}(Q) / C_{N \cap S}(Q)$ is isomorphic to $A_{5}$ or $S_{5}$. It is clear that $C_{N \cap S}(Q)=Q \times K$, where $K$ is some $\{q, 7\}^{\prime}$-subgroup. Further, the quotient group $C_{N}(Q) / K$ is a central extension of a group isomorphic to $Q$ by a group isomorphic to $A_{5}$ or $S_{5}$. Since the Schur multiplier of the group $A_{5}$ has order 2, a Sylow $q$-subgroup in $C_{N}(Q)$ is noncyclic and, consequently, contains a noncyclic subgroup $W$ of order $q^{2}$. However, $C_{P}(w) \neq 1$ for some nontrivial element $w \in W$ and, consequently, $G$ contains an element of order $7 q$, which contradicts Lemma 2.1.

The lemma is proved.

Lemma 2.4. The group $\bar{G}$ is almost simple, and the order of its socle is a multiple of 7 . 
Proof. Let $M$ be a minimal normal subgroup in $\bar{G}$. Then, $M=M_{1} \times \ldots \times M_{n}$, where $M_{1}, \ldots, M_{n}$ are isomorphic nonabelian simple groups.

Assume that $n>1$. Then, by Lemma 2.1, $7 \notin \pi(M)$, which implies by Lemma 2.2 that $M_{1}$ is isomorphic to $A_{5}, A_{6}$, or $U_{4}(2)$.

By Lemma 2.1, $7 \notin \pi(S)$ and, hence, $7 \in \pi(\bar{G} / M)$. Let $x$ be an element of order 7 in $\bar{G}$. Since the group $N_{\bar{G}}\left(M_{1}\right) / C_{\bar{G}}\left(M_{1}\right)$ is isomorphic to a subgroup of the $7^{\prime}$-group $\operatorname{Aut}\left(M_{1}\right)$, we conclude that, if the element $x$ normalizes $M_{1}$, then it centralizes $M_{1}$; consequently, $21 \in \omega(\bar{G})$, which contradicts Lemma 1.1. Therefore, the subgroup $K:=\left\langle M_{1}, x\right\rangle$ is isomorphic to the wreath product $M_{1}<7$; hence, $C_{K^{\prime}}(x) \cong M_{1}$, which implies once again that $21 \in \omega(G)$, a contradiction.

Thus, $n=1$; i.e., $M$ is simple. If, in $\bar{G}$, there exists a minimal normal subgroup $N$ different from $M$, then, as proved above, $N$ is simple and centralizes $M$. Arguing as above, we come to a contradiction.

Thus, $M$ is the simple socle of the group $\bar{G}$; i.e., $\bar{G}$ is almost simple. The second statement of the lemma follows from Lemmas 2.2 and 2.3 and from [9].

The lemma is proved.

Further, let $M:=\operatorname{Soc}(\bar{G})$.

Lemma 2.5. If $M \in\left\{L_{2}(8), L_{2}(49), A_{8}, A_{9}, S_{6}(2), O_{8}^{+}(2)\right\}$, then $S=O_{2}(G)$. In particular, $M$ is not isomorphic to $L_{2}(8)$ and $L_{2}(49)$.

Proof. Assume that the condition of the lemma is satisfied but $S \neq O_{2}(G)$. Then, in view of Lemma 2.3, $\pi(S)$ contains a number $p \in\{3,5\}$. By [9], the group $M$ contains a subgroup isomorphic either to a Frobenius group of the form $2^{3}: 7$ or to an elementary group of order 49 (for $M \cong L_{2}(49)$ ). Denote by $R$ a 2 -complement in the complete preimage of this subgroup in $G$.

Suppose that the first case holds. By Lemma 2.3, $|R|_{7}=7$; consequently, $R$ is a Frobenius group with kernel $R \cap S=F(R)=O_{3}(R) \times O_{5}(R)$ and complement of order 7 . Therefore, $N_{H}(F(R))=F(R) \lambda L$, where $L=O_{2}(L) \lambda\langle x\rangle,|x|=7$, and $\left[O_{2}(L), x\right] \neq 1$. By Proposition 1.3, $C_{F(R)}(x) \neq 1$, which contradicts Lemma 2.1.

Suppose that the second case holds. Then, $R /(R \cap S) \cong 7^{2}$; consequently, $R$ can be neither a Frobenius group nor a 2-Frobenius group, a contradiction.

Thus, $S=O_{2}(G)$. If $M$ is isomorphic to $L_{2}(8)$ or $L_{2}(49)$, then there exist no elements of order 15 in $G$, which contradicts Lemma 2.1.

The lemma is proved.

Lemma 2.6. If $S=O_{2}(G)$, then statement (4) of the theorem holds.

Proof. Assume that $G$ is a counterexample to the lemma. Then, it follows from Lemmas 2.2, 2.4, and 2.5 that $M \in\left\{L_{3}(4), U_{3}(5), U_{4}(3)\right\}$. In addition, the group $\bar{G}$ contains an element of order 15; hence, in view of [9], the group $\bar{G}$ contains an element of order 21, which contradicts Lemma 2.1.

The lemma is proved.

In view of Lemmas 2.5 and 2.6, we will assume further that $S \neq O_{2}(G)$. Then, by Lemma 2.3, $\pi(S)$ contains a number $p \in\{3,5\}$. In addition, by Lemmas 2.2, 2.4, and 2.5, the group $M$ is isomorphic to $L_{2}(7), U_{3}(3), A_{7}, L_{3}(4), U_{3}(5), U_{4}(3)$, or $J_{2}$. By [9], the group $M$ contains a subgroup isomorphic to a Frobenius group of the form $7: 3$. Suppose that $H$ is a complete preimage of this subgroup in $G$ and $R$ is a 2-complement in $H$. Then, $|R|_{7}=7$; consequently, for the solvable group $H$, statement (3) of the theorem holds. Thus, $R$ is a 2-Frobenius group of the 
form $F(R): 7: 3$, where $F(R)=R \cap S=O_{3}(R) \times O_{5}(R)$; moreover, $F(R)$ is a 2-complement in $S$ and $O_{2}(G) F(R)=O_{2,2^{\prime}}(S)$.

Without loss of generality, we assume further that $O_{2}(G)=1$. Then, $S=F(G) \lambda T$ for a Sylow 2-subgroup $T$ in $S$. By Frattini's argument, we have $G=S N_{G}(T)$; consequently, $N_{G}(T)$ contains an element $x$ of order 7. Applying Proposition 1.4 to the group $F(G) \lambda T \lambda\langle x\rangle$, we find that $T$ centralizes $x$. Therefore, $\overline{C_{G}(T)}$ contains $M$. Let $L:=C_{G}(T)^{\infty}$. Then, the quotient group $F(G) L / F(G)$ is isomorphic to the covering group $\widehat{M}$ for $M$ and, moreover, $Z(\widehat{M})$ is a 2-group.

Lemma 2.7. If $Z(\widehat{M})=1$, then one of statements (5)-(7) of the theorem holds.

Proof. Let $Z(\widehat{M})=1$. Then, the simple group $M$ acts on $F(G)$ so that $C_{F(G)}(g)=1$ for an element $g$ of order 7 from $M$. Applying Proposition 1.2 to the element $g$ and the tables of 3 -modular and 5-modular Brauer characters of the group $M$ from $[7,9]$, we obtain the statement of the lemma.

As an example, consider the case when the group $M$ is isomorphic to $L_{2}(7)$. Let $p \in\{3,5\}$. By Proposition 1.2 and the table of $p$-modular Brauer characters of the group $M$ from [7], there exist only the following absolutely irreducible $M$-modules over a field of characteristic $p$ on which the element $g$ acts freely: two 3-dimensional modules with the definition field $G F(p)(\sqrt{-7})=$ $G F\left(p^{2}\right)$ and one 6-dimensional module with the definition field $G F(p)$. Since the two 3-dimensional modules are algebraically conjugate, we conclude by [15, Theorem VII.1.16] that there exist a unique 6-dimensional irreducible but not absolutely irreducible $G F(p) M$-module (it is isomorphic to a 3-dimensional $G F\left(p^{2}\right) M$-module considered over the field $\left.G F(p)\right)$ and a unique 6-dimensional absolutely irreducible $G F(p) M$-module.

The lemma is proved.

Now, let $Z(\widehat{M}) \neq 1$. Then, in view of [9], the group $\widehat{M}$ is isomorphic to $S L_{2}(7), Z \cdot L_{3}(4)$ with the group $Z$ isomorphic to a nontrivial subgroup from $4 \times 4,2 \cdot U_{4}(3) \cong \Omega_{6}^{-}(3), 4 \cdot U_{4}(3) \cong S U_{4}(3)$, or $2 J_{2}$. The quasi-simple group $\widehat{M}$ acts faithfully on $F(G)$ so that $C_{F(G)}(x)=1$ for an element $x$ of order 7 from $\widehat{M}$. By Lemma 2.7, we can assume that $C_{F(G)}(Z(\widehat{M})=1$. Using Proposition 1.2 and the tables of 3-modular and 5-modular Brauer characters of $\widehat{M}$ from [7,9], we find that one of statements (8)-(11) of the theorem holds.

The theorem is proved.

\section{PROOF OF THE COROLLARY}

Let $G$ be a finite group such that $|G|=\left|J_{2}\right|$ and $\Gamma(G)=\Gamma$ (Aut $\left.\left(J_{2}\right)\right)$. Then, $|G|=2^{8} \times 3^{3} \times 5^{2} \times 7$.

Let $G$ be solvable. Then, a 2-complement in $G$ has order $3^{3} \times 5^{2} \times 7$; hence, according to the theorem, it is either a Frobenius group of the form $\left(3^{3} \times 5^{2}\right): 7$ or a 2-Frobenius group of the form $\left(3^{3} \times 5^{2}\right):(7: 3)$. This is impossible, since 7 does not divide $5^{2}-1$.

Thus, $G$ is unsolvable. In view of the theorem, the group $\bar{G}=G / S(G)$ is almost simple with socle $M$ isomorphic to $L_{2}(7), U_{3}(3), A_{7}, A_{8}, A_{9}, S_{6}(2), O_{8}^{+}(2), L_{3}(4), U_{4}(3)$, or $J_{2}$. Moreover, the quotient group $\bar{G} / M$ is a 2-group.

If $M \in\left\{A_{8}, A_{9}, S_{6}(2), O_{8}^{+}(2)\right\}$, then $S(G)=O_{2}(G)$, which contradicts the equalities $\left|A_{8}\right|_{5}=5$, $\left|A_{9}\right|_{3}=3^{4},\left|S_{6}(2)\right|_{2}=2^{9}$, and $\left|O_{8}^{+}(2)\right|_{2}=2^{12}$.

If $M \cong U_{4}(3)$, then $|M|_{3}=3^{6}$, a contradiction with the fact that $|G|_{3}=3^{3}$.

If $M \cong A_{7}$, then $|M|_{3}=3$; consequently, $21 \in \omega(G)$, a contradiction with Lemma 2.1.

If $M \in\left\{L_{2}(7), U_{3}(3)\right\}$, then $|S(G)|_{5}=5^{2}$; consequently, $35 \in \omega(G)$, a contradiction with Lemma 2.1. 
If $M \cong L_{3}(4)$, then $|S(G)|_{5}=5$; consequently, $35 \in \omega(G)$, a contradiction with Lemma 2.1.

Thus, $M \cong J_{2}$. If $\bar{G} \cong \operatorname{Aut}\left(J_{2}\right)$, then $G \cong \operatorname{Aut}\left(J_{2}\right)$. If $\bar{G} \cong J_{2}$, then $|S(G)|=2$; consequently, $G$ is isomorphic to $2 \times J_{2}$ or $\widehat{J_{2}}$.

The corollary is proved.

\section{ACKNOWLEDGMENTS}

This work was supported by the Russian Foundation for Basic Research (project no. 10-0100324), by a joint grant of the Russian Foundation for Basic Research and the National Natural Science Foundation of China (project no. 12-01-91155), by a Program of the Division of Mathematical Sciences of the Russian Academy of Sciences (project no. 12-T-1-1003), and by Programs of Joint Research of the Ural Branch of the Russian Academy of Sciences with the Siberian Branch of the Russian Academy of Sciences (project no. 12-S-1-1018) and the Belorussian National Academy of Sciences (project no. 12-S-1-1009).

\section{REFERENCES}

1. A. V. Vasil'ev, M. A. Grechkoseeva, and V. D. Mazurov, "Characterization of finite simple groups by spectrum and order," Algebra Logic 48 (6), 385-409 (2009).

2. S. Dolfi, E. Jabara, and M. S. Lucido, "C55-groups," Sib. Math. J. 45 (6), 1053-1062 (2004).

3. A. S. Kondrat'ev and I. V. Khramtsov, "On Finite Tetraprimary Groups," Proc. Steklov Inst. Math. 279 (Suppl. 1), S43-S61 (2012).

4. C. Curtis and I. Reiner, Representation Theory of Finite Groups and Associative Algebras (Wiley, New York, 1962; Nauka, Moscow, 1969).

5. V. D. Mazurov, "Characterizations of finite groups by sets of the orders of their elements," Algebra Logic 36 (1), 23-32 (1997).

6. V. D. Mazurov, "Groups with specified spectrum," Izv. Ural'sk. Gos. Univ. 36, 119-138 (2005).

7. C. Jansen, K. Lux, R. Parker, and R. Wilson, An Atlas of Brauer Characters (Clarendon, Oxford, 1995).

8. M. Aschbacher, Finite Group Theory (Cambridge Univ. Press, Cambridge, 1986).

9. J. H. Conway, R. T. Curtis, S. P. Norton, et al., Atlas of Finite Groups (Clarendon, Oxford, 1985).

10. Y. Bugeaud, Z. Cao, and M. Mignotte, "On simple $K_{4}$-groups," J. Algebra 241 (2), 658-668 (2001).

11. G. Chen, "A new characterization of sporadic simple groups," Algebra Colloq. 3 (1), 49-58 (1996).

12. D. Gorenstein, Finite Groups (Harper and Row, New York, 1968).

13. M. Hagie, "The prime graph of a sporadic simple group," Comm. Algebra 31 (9), 4405-4424 (2003).

14. M. Herzog, "On finite simple groups of order divisible by three primes only," J. Algebra 10 (3), 383-388 (1968).

15. B. Huppert and N. Blackburn, Finite Groups II (Springer-Verlag, Berlin, 1982).

16. B. Khosravi, "On the prime graph of the automorphism groups of sporadic simple groups," Arch. Math. (Brno) 45 (2), 83-94 (2009).

17. B. Khosravi, "A characterization of the automorphism groups of sporadic groups by the set of orders of maximal abelian subgroups," Kumamoto J. Math. 22, 17-34 (2009).

18. B. Khosravi, "On the prime graph of a finite group," in Groups St Andrews 2009 in Bath (Cambridge, Cambridge Univ. Press, 2011), Ser. London Math. Soc. Lecture Notes, Vol. 388, Issue 2, pp. 424-428.

19. W. J. Shi, "On simple $K_{4}$-groups," Chinese Science Bull. 36 (17), 1281-1283 (1991).

20. J. S. Williams, "Prime graph components of finite groups," J. Algebra 69 (2), 487-513 (1981).

Translated by E. Vasil'eva 